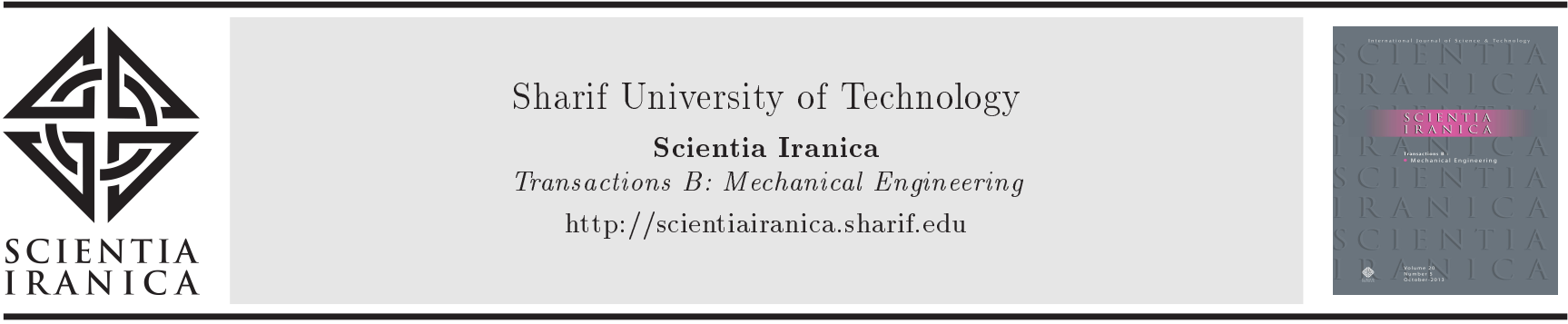

\title{
Pull-in criteria of a non-classical microbeam under electric field using homotopy method
}

\author{
R. Derakhshan ${ }^{a}$, M.T. Ahmadian ${ }^{\text {,**, }}$, and K. Firoozbakhsh ${ }^{\mathrm{a}}$ \\ a. School of Mechanical Engineering, Sharif University of Technology, Tehran, P.O. Box 11155-9567, Iran. \\ b. Center of Excellence in Design, Robotic and Automation, Member of School of Mechanical Engineering, Member of USERN, \\ Sharif University of Technology, Tehran, P.O. Box 11155-9567, Iran.
}

Received 9 May 2015; received in revised form 19 September 2016; accepted 31 May 2017

\section{KEYWORDS \\ Microbeam; \\ Pull-in; \\ Strain gradient \\ theory; \\ HAM.}

\begin{abstract}
In this study, a homotopy analysis method was used to obtain analytic solutions to predict dynamic pull-in instability of an electrostatically-actuated microbeam. The nonlinear describing equation of a microbeam affected by an electric field, including the fringing field effect, was obtained based on strain gradient elasticity, couple stress, and classical theory. Influences of different parameters on dynamic pull-in instability were investigated. The equation of motion of a double-clamped microbeam was discretized and solved by using Galerkin's method via mode summation. The resulting non-linear differential equation was also solved by using the Homotopy Analysis Method (HAM). The influence of HAM parameters on accuracy was studied specifically in the vicinity of the pull-in voltage. Comparison of the results of pull-in voltage indicated that low-voltage good agreement exists between numerical and semi-analytical methods, while HAM results deviated from those of numerical methods at high voltages. Findings indicate that strain gradient and couple stress effects result in a stiffer microbeam than with classical theory. Effects of an auxiliary parameter on convergence were also studied. Convergence domains were determined at different voltages and orders of HAM approximation.
\end{abstract}

(C) 2018 Sharif University of Technology. All rights reserved.

\section{Introduction}

A microbeam, actuated by electrostatic distributed force, is a flexible beam-shaped element attached to a rigid substrate, as illustrated in Figure 1. Electrostatically-actuated microbeams are extensively used in different applications such as signal filtering and mass sensing [1]. When the input voltage exceeds

\footnotetext{
*. Corresponding author.

E-mail addresses: rezaderakhshan@mech.sharif.edu ( $R$. Derakhshan); ahmadian@sharif.edu (M.T. Ahmadian); firoozbakhsh@sharif.edu (K. Firoozbakhsh).
}

doi: $10.24200 /$ sci. 2017.4315 a critical value, called the pull-in voltage $\left(V_{p i}\right)$, the flexible microbeam spontaneously deflects towards the rigid plate. Pull-in instability is a basic phenomenon considered in the design of the micro actuators. Pull-in instability was observed experimentally by Taylor [2] and Nathanson et al. [3]. Osterberg studied Micro Electro Mechanical Systems (MEMS) with circular and rectangular shapes and achieved several closed-form models for pull-in instability in these systems [4].

When the rate of voltage variation is low and, consequently, inertia has almost no influence on the microsystem behavior, the critical value of voltage is called the static pull-in voltage $\left(V_{p i}\right)$. However, when the rate of voltage variation is not negligible, the effect of inertia has to be considered and the critical voltage 
value is called the dynamic pull-in voltage $\left(V_{p i d}\right)$. The pull-in instability related to this situation is called the dynamic pull-in instability $[5,6]$.

Studying vibrational behavior of MEMS is quite useful in determining design parameters of these systems. Vibrational characteristics of microbeams have been generally studied assuming small vibrations around a deflected position. In [7], oscillatory behavior of microbeams considering mid-plane stretching has been studied. In [1], vibrations of double-clamped microbeams, under an electric field, have been investigated. Vibrations of electrostatically actuated microstructures have also been studied in [8-10].

Since the classical continuum theory can neither capture the size-dependency observed in micro-scale components nor accurately predict the mechanical behavior of such components, non-classical continuum theory has been developed. The couple stress theory, a powerful non-classical continuum theory, was introduced and elaborated by Koiter [11] and by Mindlin and Tiersten [12] in the early 1960s. The additional material parameters appearing in this theory (in addition to the two classical Lamé parameters, elastic modulus, and Poisson's ratio) enable the theory not only to capture the size-dependency, but also to bring about higher accuracy in modeling of the micro-scale structures. A Timoshenko beam model was developed based on this theory by Asghari et al. [13]. The size-dependent static behavior of this new beam model has been investigated, and it is observed that the bending stiffness of the new model is greater than that of the classical Timoshenko beam model. Yang et al. [14] introduced the modified couple stress theory by modifying the couple stress continuum theory. He utilized the equilibrium equation of moments of couple in addition to the two conventional equilibrium equations, i.e. equilibrium equation of forces and moment of forces. Soon after that, the modified couple stress theory became a popular non-classical theory to develop microbeam and microplate models as well as to deal with the size-dependent problems in MEMS [15-19].

The strain gradient elasticity theory was originally developed by Mindlin [20-22]. Wang et al. [23] used the strain gradient theory and took the Timoshenko beam model to study dynamic microbeams. Sedighi [24] also used this theory and took the EulerBernoulli beam to study dynamical characteristics of microbeams.

Different techniques have been proposed for finding solutions to nonlinear equations of MEMS: the differential quadrature method [25], the finite-element method $[9,10]$, and homotopy methods [26]. Although it is difficult to get an analytic approximation for different phenomena in MEMS, there are some analytic methods for nonlinear problems of microelectromechanical systems such as perturbation tech- niques $[27,28]$. In general, perturbation approximations are valid only for weakly nonlinear problems [29]. Based on the homotopy method in topology, Liao proposed the Homotopy Analysis Method (HAM) to present analytic solutions to strongly nonlinear problems [29]. This method can also be effective in finding solutions to the vibrations of microbeams. In numerical analysis, the Runge-Kutta methods are a family of implicit and explicit iterative methods used in temporal discretization for the approximate solutions to ordinary differential equations [30].

\section{Modeling and formulation}

A clamped-clamped microbeam with a rigid substrate under electrostatic actuated voltage, $\mathrm{V}$, is represented in Figure 1.

The microbeam is of length, $L$, thickness, $h$, width, $b$, density, $\rho$, moment of inertia, $I$, modulus of elasticity, $E$, and initial air gap, $d$. The equation of motion for a microactuated beam based on strain gradient elasticity theory and considering the EulerBernoulli beam is [24,31]:

$$
\begin{aligned}
& \rho A_{0} \frac{d^{2}}{d t^{2}} w(x, t)+\frac{\hat{\alpha} b^{3}}{\left(d-w(x, t)+6 \lambda_{0}\right)(d-w(x, t))^{2}} \\
& \frac{d}{d t} w(x, t)-\frac{2}{5} \mu l\left(5 l_{0}{ }^{2}+2 l_{1}{ }^{2}\right) \frac{d^{6}}{d x^{6}} w(x, t) \\
& +\left[E I+\frac{1}{15} \mu A_{0}\left(30 l_{0}^{2}+8{l_{1}}^{2}+15 l_{2}{ }^{2}\right)\right] \frac{d^{4}}{d x^{4}} w(x, t) \\
& -\left(N+\frac{E b h}{2 L} \int_{0}^{L}\left(\frac{d}{d x} w(x, t)\right)^{2} d x\right) \frac{d^{2}}{d x^{2}} w(x, t) \\
& -F_{\text {es }}=0 .
\end{aligned}
$$

Here $\mu, A_{0}$, and $N$ represent shear modulus, crosssection area of microbeam, and initial axial load, respectively, with $l_{0}, l_{1}$, and $l_{2}$ denoting the additional independent material length scale parameters associated with dilatation gradients, deviatoric stretch gradients, and symmetric rotation gradients, respectively [32]. $N$ is positive in tension and negative in compression.

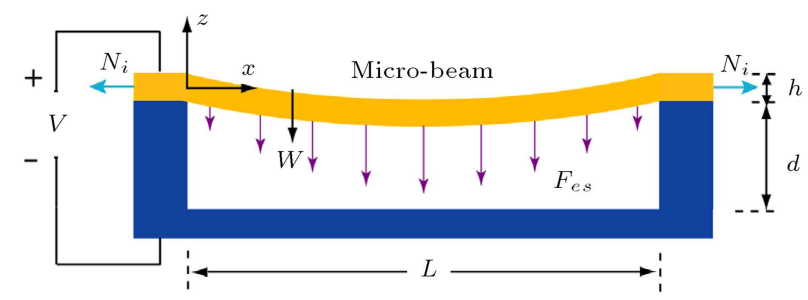

Figure 1. The configuration of an electrostatically actuated microbeam [24]. 
The second term in Eq. (1) represents the effect of damping force of the fluid that exists in the air gap. $\hat{\alpha}$ is the viscosity of the fluid and $\lambda_{0}$ is the average distance between the molecules of the fluid.

A uniform electric field does not drop suddenly to zero at edges. Actually, a fringing field always exists, and a more realistic situation, including the "fringing-field", should be taken into consideration. The electrostatic force per unit length can be written as follows $[1,33]$ :

$$
F_{e s}=\frac{1}{2} \frac{b \varepsilon V^{2}\left(1+f_{f}\right)}{(d-w(x, t))^{2}},
$$

$\varepsilon=8.854187817620 \times 10^{-12}(\mathrm{~F} / \mathrm{m})$ is the vacuum permittivity. The first term in parentheses in the numerator of Eq. (2) describes the parallel-plate approximation; the second one, $f_{f}$, accounts for the fringing fields effect due to the finite width where:

$$
f_{f}=\frac{\beta(d-w(x, t))}{b} .
$$

For a double clamped beam, $\beta$ is set to $0.65[34,35]$. The use of the following non-dimensional parameters:

$$
\begin{aligned}
& W(\xi, \tau)=\frac{w(x, t)}{d}, \quad \tau=\sqrt{\frac{I E}{\rho b h L^{4}}} t, \quad \xi=\frac{x}{L}, \\
& \alpha=\frac{6 d^{2}}{h^{2}}, \quad \lambda^{2}=\frac{24 \varepsilon L^{4} V^{2}}{E h^{3} d^{3}}, \quad f_{i}=\frac{N L^{2}}{E I}, \quad \gamma=\frac{d}{b}, \\
& \mu_{s}=\frac{12 \mu l_{2}^{2}}{E h^{2}}, \quad K_{n_{0}}=\frac{\lambda_{0}}{d}, \quad \hat{c}=\frac{\hat{\alpha} b^{3} L^{2}}{\sqrt{E I \rho A_{0}}} .
\end{aligned}
$$

recasts Eq. (1) into the non-dimensional form as follows:

$$
\begin{gathered}
\frac{\partial^{2}}{\partial \tau^{2}} W(\xi, \tau)+\frac{\hat{c}}{\left(1-W(\xi, \tau)+6 K_{n_{0}}\right)(1-W(\xi, \tau))^{2}} \\
\left(\frac{\partial}{\partial \tau} W(\xi, \tau)\right)-\frac{1}{30} \frac{\mu_{s}\left(\frac{5 l_{0}{ }^{2}}{l_{2}{ }^{2}}+\frac{2 l_{1}^{2}}{l_{2}{ }^{2}}\right) h^{2}}{L^{2}}\left(\frac{\partial^{6}}{\partial \xi^{6}} W(\xi, \tau)\right) \\
+\left[1+\frac{1}{15} \mu_{s}\left(\frac{30 l_{0}^{2}}{l_{2}^{2}}+\frac{l_{1}^{2}}{l_{2}^{2}}+15\right)\right]\left(\frac{\partial^{4}}{\partial \xi^{4}} W(\xi, \tau)\right) \\
-\left[f_{i}+\alpha\left(\int_{0}^{1}\left(\frac{\partial}{\partial \xi} W(\xi, \tau)\right) d \xi\right)\right]\left(\frac{\partial^{2}}{\partial \xi^{2}} W(\xi, \tau)\right) \\
-\frac{1}{4} \frac{\lambda^{2}(1+\gamma \beta(1-W(\xi, \tau)))}{(1-W(\xi, \tau))^{2}}=0 .
\end{gathered}
$$

Here, $W(\xi, \tau), \quad \frac{\partial}{\partial \tau} W(\xi, \tau), \frac{\partial^{2}}{\partial \tau^{2}} W(\xi, \tau)$, and $\tau$ are normalized deflection, normalized velocity, normalized acceleration, and normalized time, respectively. Usually, microbeams work in vacuum because the damping causes energy loses. So, by considering a Taylor expansion for electrostatic force and in the absence of damping, Eq. (5) can be written as follows:

$$
\begin{gathered}
\frac{\partial^{2}}{\partial \tau^{2}} W(\xi, \tau)+\left[1+\frac{1}{15} \mu_{s}\left(\frac{30 l_{0}^{2}}{l_{2}^{2}}+\frac{l_{1}^{2}}{l_{2}^{2}}+15\right)\right] \\
\left(\frac{\partial^{4}}{\partial \xi^{4}} W(\xi, \tau)\right)-\frac{1}{30} \frac{\mu_{s}\left(\frac{5 l_{0}^{2}}{l_{2}^{2}}+\frac{2 l_{1}^{2}}{l_{2}^{2}}\right)\left(\frac{\partial^{6}}{\partial \xi^{6}} W(\xi, \tau)\right) h^{2}}{L^{2}} \\
-\left[f_{i}+\alpha\left(\int_{0}^{1}\left(\frac{\partial}{\partial \xi} W(\xi, \tau)\right)^{2} d \xi\right)\right]\left(\frac{\partial^{2}}{\partial \xi^{2}} W(\xi, \tau)\right) \\
-\frac{\lambda^{2}}{4} \sum_{j=0}^{m}(j+1+\gamma \beta)(W(\xi, \tau))^{j}=0 .
\end{gathered}
$$

In general, one can assume that:

$$
W(\xi, \tau)=\sum_{r=1}^{n} \mathrm{u}_{r}(\tau) \phi_{r}(\xi),
$$

where $r$ is the number of modes included in the simulation, $n$ is the number of degrees of freedom, $m$ is the number of the electrostatic force Taylor approximation, and $\phi_{r}(\xi)$ represents the mode shapes of clampedclamped microbeam. Assuming a first approximation for $\phi_{1}(\xi)$ we have:

$$
\begin{aligned}
\phi_{1}(\xi) & =\cosh \left(\lambda_{1} \xi\right)-\cos \left(\lambda_{1} \xi\right) \\
& -\frac{\cosh \left(\lambda_{1}\right)-\cos \left(\lambda_{1}\right)}{\sinh \left(\lambda_{1}\right)-\sin \left(\lambda_{1}\right)}\left(\sinh \left(\lambda_{1} \xi\right)-\sin \left(\lambda_{1} \xi\right)\right)
\end{aligned}
$$

where $\lambda_{1}=4.730040745$ is the first root of characteristic equation of clamped-clamped beam. Using Galerkin's decomposition method [30], the nonlinear governing equation of motion according to dimensionless time can be written as follows:

$$
\begin{aligned}
\frac{d^{2}}{d \tau^{2}} u(\tau) & +M_{0}+M_{1} u(\tau)+M_{2} u(\tau)^{2}+M_{3} u(\tau)^{3} \\
& +M_{4} u(\tau)^{4}+M_{5} u(\tau)^{5}+M_{6} u(\tau)^{6}=0
\end{aligned}
$$

in which $M_{0}$ to $M_{6}$ are constants (see Appendix A).

Eq. (9) is an Ordinary Differential Equation (ODE) with sixth degrees $(m=6)$ of Taylor approximation for the electrostatic force solvable by HAM.

When $l_{0}=l_{1}=0$, results approach those of couple stress theory; when $l_{0}=l_{1}=l_{2}=0$, results approach those of classical theory. Also, Eq. (9) was solved by a Runge-Kutta numerical method whose results were used to validate results obtained by the HAM method. 


\section{Application of homotopy analysis method to microbeam dynamic characteristics}

The Homotopy Analysis Method (HAM) is an effective analytic method for solving nonlinear equations. This method transforms a general nonlinear problem into an infinite number of linear problems by embedding an auxiliary parameter, $q[36]$. As $q$ increases from 0 to 1 , the solution varies from the initial approximation to the exact solution. Homotopy function, $\mathcal{H}(\Phi ; q, \hbar, H(\tau))$, is constructed as $[29,36-43]$ :

$$
\begin{aligned}
& \mathcal{H}(\Phi ; q, \hbar, H(\tau))=(1-q) \mathcal{L}\left[\Phi(\tau ; q)-u_{0}(\tau)\right] \\
& \quad-q \hbar H(\tau) \mathfrak{N}[\Phi(\tau ; q), \Lambda(q)]
\end{aligned}
$$

where $\hbar, u_{0}(\tau), H(\tau), \mathcal{L}$, and $\mathfrak{N}$ are a nonzero auxiliary parameter, an initial guess, a nonzero auxiliary function, an auxiliary linear operator, and a nonlinear operator, respectively. Values of $\hbar$ and $H(\tau)$ adjust the convergence region of the solution. For the microbeam problem, the auxiliary function can be chosen in the form of $H(\tau)=1$.

From Eq. (9), the nonlinear operator can be expressed as follows:

$$
\begin{gathered}
\mathfrak{N}(\Phi(\tau ; q), \Lambda(q))=\frac{\partial^{2}}{\partial \tau^{2}} \Phi(\tau ; q)+\Lambda(q) \Phi(\tau ; q)+M_{0} \\
+M_{2} \Phi(\tau ; q)^{2}+M_{3} \Phi(\tau ; q)^{3}+M_{4} \Phi(\tau ; q)^{4} \\
+M_{5} \Phi(\tau ; q)^{5}+M_{6} \Phi(\tau ; q)^{6}
\end{gathered}
$$

subject to initial conditions of zero and with $\Lambda(1)=$ $M_{1}$.

Moreover, the auxiliary linear operator can be defined as follows:

$$
\mathcal{L}(\Phi(\tau ; q))=\frac{\partial^{2}}{\partial \tau^{2}} \Phi(\tau ; q)+\omega^{2} \Phi(\tau ; q),
$$

function $\Phi(\tau ; q)$ can be expanded in a power series of embedding parameter $q$ using Taylor's theorem as follows:

$$
\Phi(\tau ; q)=\sum_{i=0}^{\infty} q^{i} u_{i}(\tau) .
$$

Also:

$$
\Lambda(q)=\omega^{2}+\sum_{i=0}^{\infty} q^{i} \omega_{i}(\omega)
$$

By equating to zero the homotopy function, the zeroorder deformation equation is constructed as follows:

$$
(1-q) \mathcal{L}\left[\Phi(\tau ; q)-u_{0}(\tau)\right]=q \hbar \mathfrak{N}[\Phi(\tau: q),(\Lambda q)]
$$

$$
\begin{aligned}
& \Phi(0 ; q)=0, \\
& \frac{d \Phi(0 ; q)}{d \tau}=0 .
\end{aligned}
$$

When $q=0$, Eq. (15) becomes:

$$
\mathcal{L}\left[\Phi(\tau ; 0)-u_{0}(\tau)\right]=0,
$$

which gives the zero-order approximation of $u(\tau)$. It is straightforward to set the initial guess $u_{0}(\tau)$ to zero. Differentiating Eq. (15) with respect to $q$ and then setting $q=0$ yields the first-order deformation equation, which gives the first-order approximation of $\mathrm{u}(\tau)$ by solving:

$$
\mathcal{L}\left[u_{l}(\tau)\right]=\hbar \mathfrak{N}[\Phi(0 ; q), \Lambda(q)] .
$$

Subject to zero initial conditions, the higher-order approximations of solution $u(t)$ can be found by solving high-order deformation equations. After differentiating Eq. (15) $j$ times with respect to $q$, then setting $q=0$, and finally dividing each side by $j$ !, one can obtain the $j$ th-order deformation equation:

$$
\begin{aligned}
\mathcal{L}\left[u_{j}(\tau)-\chi_{j} u_{j-1}(\tau)\right]= & \\
& \left.\frac{1}{(j-1) !} \hbar \frac{\partial^{j-1} \mathfrak{N}[\Phi(\tau ; q), \Lambda(q)]}{\partial q^{j-1}}\right|_{q=0},
\end{aligned}
$$

where,

$$
\chi_{j}= \begin{cases}0 & \text { when } j \leq 1 \\ 1 & \text { otherwise }\end{cases}
$$

It is noteworthy that the vibrations of an undamped microbeam under the actuation of the electrostatic force can be expressed by the base functions:

$$
\cos (k \omega \tau), \quad k=1,2,3, \ldots
$$

Therefore, to eliminate the secular terms in the $j$ thorder approximation, one can set the coefficient of $\cos (\omega \tau)$ in the $(j-1)$ th-order deformation equation to zero.

After this operation, an algebraic equation is obtained from which $\omega_{j-2}$ versus $\omega$ is obtained. After finding an adequate approximation, by setting $q=1$, Eqs. (22) and (23) are obtained as follows:

$$
\begin{aligned}
& \Lambda(1)=\omega^{2}+\omega_{1}(\omega)+\omega_{2}(\omega)+\ldots+\omega_{p}(\omega)=M_{1}, \\
& u(\tau)=u_{1}(\tau)+u_{2}(\tau)+\ldots+u_{p+2}(\tau),
\end{aligned}
$$

where $p$ is the order of the electrostatic force Taylor approximation. 
The normalized frequency $(\omega)$ is only an unknown parameter in Eq. (22) and is determined by this equation.

The value of normalized frequency is determined by Eq. (22). The actual frequency $\left(\omega_{a}\right)$ can be obtained by the equation:

$$
\omega_{a}=\sqrt{\frac{I E}{\rho b h L^{4}}} \omega .
$$

\section{Results and discussions}

To discuss the results, the Young's modulus for the microbeams is assumed to be $E_{y}=166 \mathrm{GPa}$. Furthermore, it is assumed that the residual axial load, thickness, width, air initial gap, and density are: $N=$ $9 \times 10^{-4} \mathrm{~N}, h=1.5 \times 10^{-6} \mathrm{~m}, b=100 \times 10^{-6} \mathrm{~m}, d=$ $1.18 \times 10^{-6} \mathrm{~m}$ and $\rho=2329 \mathrm{~kg} / \mathrm{m}^{3}$, respectively. The residual stress may be induced during the fabrication process. The length scales are $l_{0}=l_{1}=l_{2}=0.32 \times$ $10^{-6} \mathrm{~m}$.

A comparison of natural frequency for two microbeams between the present study and previous studies is shown in Table 1. It can be seen that there is good agreement between the results.

The midpoint deflection of the double-clamped microbeam with length $L=210 \mu \mathrm{m}$ for input voltage of $25 \mathrm{~V}$ is shown in Figure 2(a). The midpoint deflection for input voltage of $30 \mathrm{~V}$ is depicted in Figure 2(b). It can be observed at low voltages that small orders of approximation $(p)$ in homotopy scale are sufficient to find the deflections accurately. Nevertheless, for high voltages, higher orders of approximations are required as discussed later.

The midpoint deflection time history of a microbeam with length $L=510 \mu \mathrm{m}$ for input voltages of 5.5 and $6.2 \mathrm{~V}$ is shown in Figure 3(a) and 3(b), respectively.

It can be observed from Figures 2 and 3 that there is excellent agreement between results of the RungeKutta method and those of the semi-analytic method (particularly, for low voltages).

When input voltage is chosen to be close to the pull-in value, the difference between the numerical and semi-analytical approaches for low approximation increases drastically. The midpoint deflection time history of a microbeam with length $L=510 \mu \mathrm{m}$ by considering strain gradient theory, using Runge-Kutta

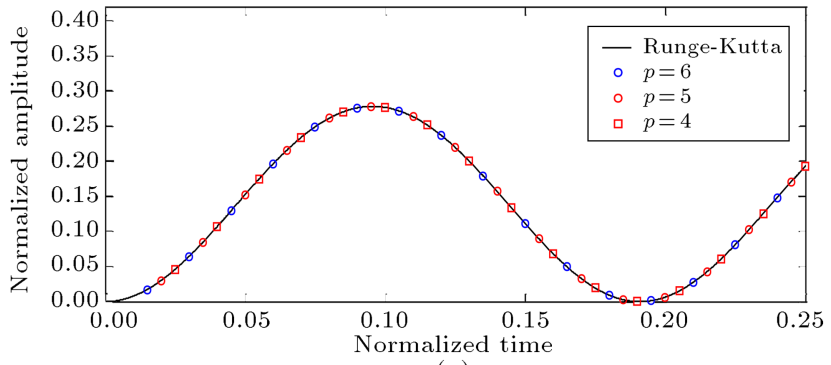

(a)

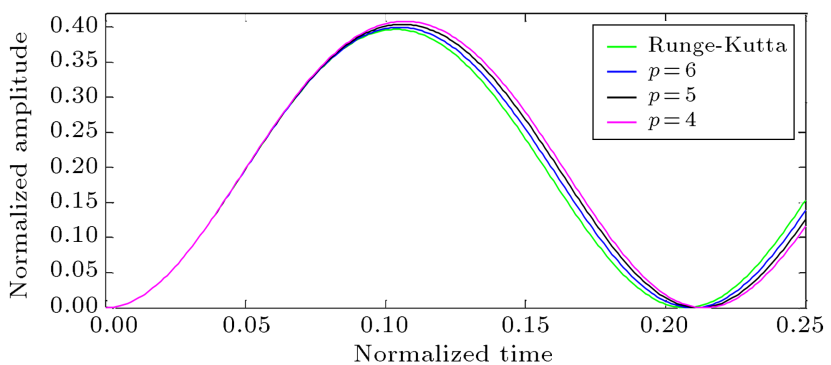

(b)

Figure 2. Midpoint-deflection-time history for the double-clamped microbeam with length $L=210 \mu \mathrm{m}$ by applying strain gradient theory: (a) Input voltage of $25 \mathrm{~V}$, and (b) input voltage of $30 \mathrm{~V}\left(V_{\text {pid }}=32.08 \mathrm{~V}\right)$, $N=9 \times 10^{-4} \mathrm{~N}$.

and semi-analytical methods for voltages close to pullin values, is shown in Figure 4. It should be noted that the Runge-Kutta method is very sensitive to input voltage, while HAM cannot recognize the small voltage variation.

Comparisons made between Figures 3 and 4 show that the nonlinear electrical force is low for low input voltages. But, by increasing input voltage to be close to pull-in value, the nonlinear force is larger and the oscillation has a non-sinusoidal shape.

Variations of normalized nonlinear frequency with applied step voltage (V) for the double-clamped microbeam with length $L=510 \mu \mathrm{m}$ for different values of $p$, using strain gradient theory, are shown in Figure 5. This diagram signifies that by increasing the voltage close to pull-in voltage, the difference between results grows. In some voltages, there are several answers for frequency in some approximations $(p)$; the true one is determined by comparing numerical results.

Variations of nonlinear frequency versus applied voltage obtained by using semi-analytic method for $p=5$ and 6 for different theories are shown in Figure 6 . As input voltage increases, the nonlinear frequency of vibrations decreases. This diagram suggests that for

Table 1. A comparison of natural frequency $(\mathrm{kHz})$ between different methods.

\begin{tabular}{cccccc}
\hline $\begin{array}{c}\text { Length } \\
(\boldsymbol{\mu} \mathbf{m})\end{array}$ & $\begin{array}{c}\text { Measured } \\
{[\mathbf{4 3}]}\end{array}$ & $\begin{array}{c}\text { Calculated } \\
{[\mathbf{4 4}]}\end{array}$ & $\begin{array}{c}\text { Calculated } \\
{[\mathbf{4 5}]}\end{array}$ & $\begin{array}{c}\text { Calculated } \\
{[\mathbf{4 6}]}\end{array}$ & $\begin{array}{c}\text { Present study } \\
\text { (HAM) }\end{array}$ \\
\hline 210 & 322.05 & 324.70 & 324.70 & 324.78 & 325.01 \\
510 & 73.79 & 74.80 & 73.46 & 74.38 & 74.74 \\
\hline
\end{tabular}




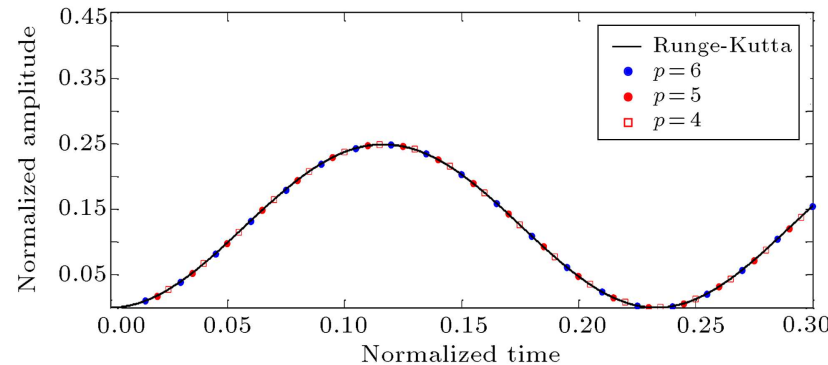

(a)

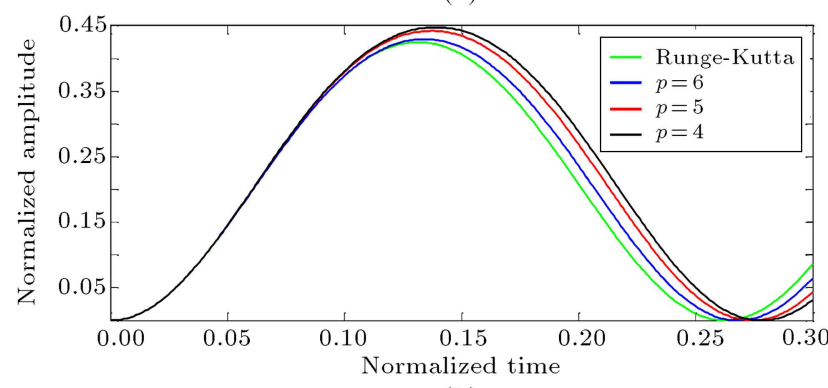

(b)

Figure 3. Midpoint deflection time history for the double-clamped microbeam with length $L=510 \mu \mathrm{m}$ by applying strain gradient theory: (a) Input voltage of $5.5 \mathrm{~V}$, and (b) input voltage of $6.2 \mathrm{~V}\left(V_{\text {pid }}=6.585 \mathrm{~V}\right)$, $N=9 \times 10^{-4} \mathrm{~N}$.

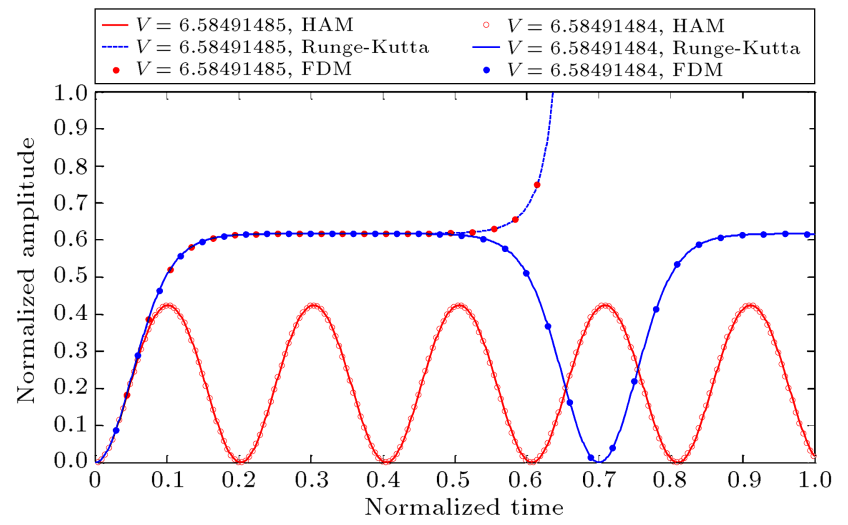

Figure 4. Midpoint-deflection-time history for the double-clamped microbeam with length $L=510 \mu \mathrm{m}$ $\left(p=6, m=6, V_{p}=6.58491485\right)$.

a large range of applied voltages, there exists good agreement between the results calculated by assuming $p=5$ and 6 in different theories. Also, the higher approximations of $p=5$ and 6 are required for accuracy.

The phase diagram of a microbeam with length $L=510 \mu \mathrm{m}$ obtained by using two methods and the strain gradient theory with zero initial conditions are shown in Figure 7. At lower voltages, there is good agreement between the two methods in various theories.

Variations of nonlinear frequency versus applied voltage using semi-analytic $(p=5,6)$ and numerical methods implementing strain gradient theory for mi-

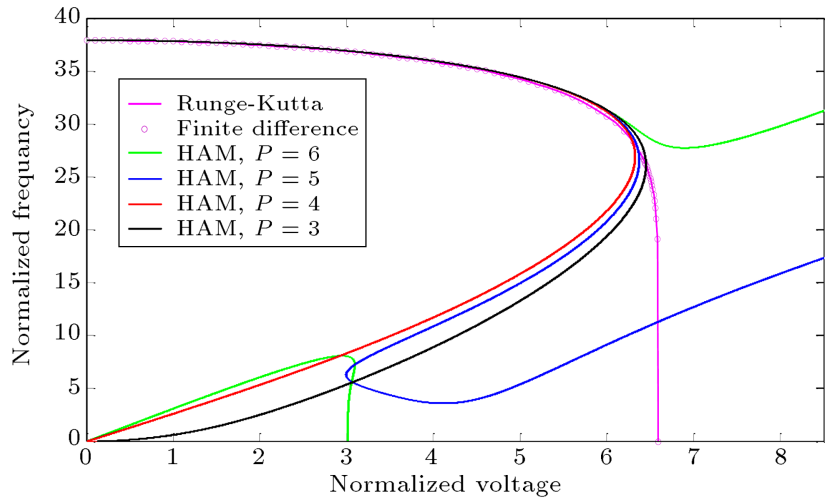

Figure 5. Variations of normalized nonlinear frequency with applied step voltage $(\mathrm{V})$ for the double-clamped microbeam with length $L=510 \mu \mathrm{m}$ and different values of $p$ with strain gradient theory.

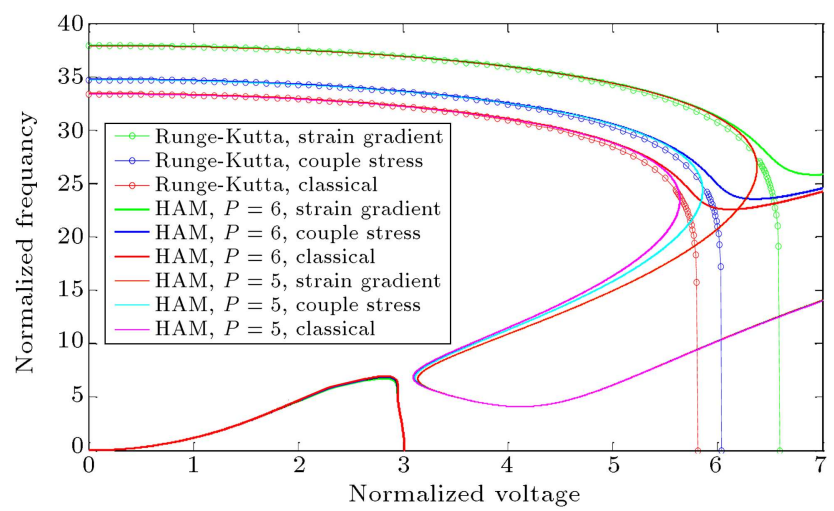

Figure 6. Variations of normalized nonlinear frequency with applied step voltage (V) for the double-clamped microbeam with length $L=510 \mu \mathrm{m}$ and different theories.

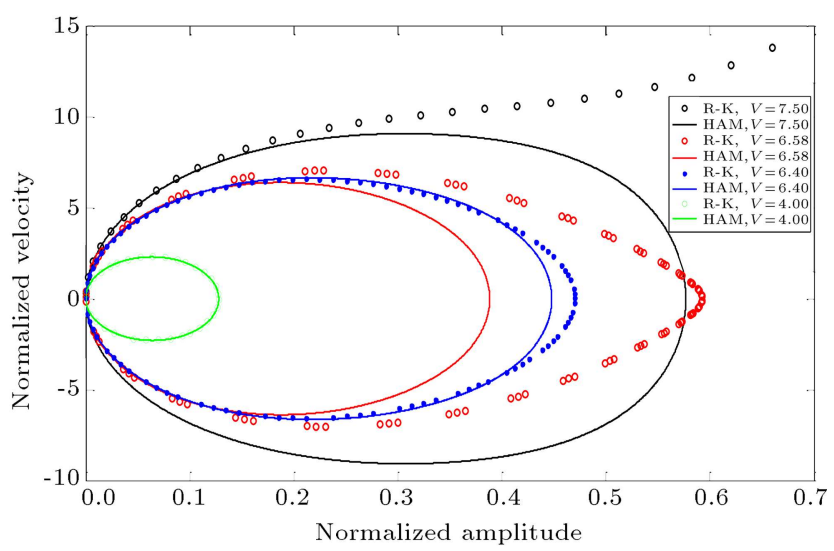

Figure 7. Phase portrait of actuated microbeam with $L=510 \mu \mathrm{m}$ based on the strain gradient elasticity theory for zero initial condition and different suddenly applied step voltages.

crobeams with length $L=510 \mu \mathrm{m}$ and $210 \mu \mathrm{m}$ are shown in Figure 8.

Midpoint-deflection-time histories of the microbeam with length $210 \mu \mathrm{m}$ for different axial loads 
are shown in Figure 9. As expected, increasing compression force enhances the deflection of the beam.

The midpoint-deflection-time history of the microbeam with length $L=210 \mu \mathrm{m}$ under step voltages of 15 and $25 \mathrm{~V}$ is shown in Figure 10. Results indicate

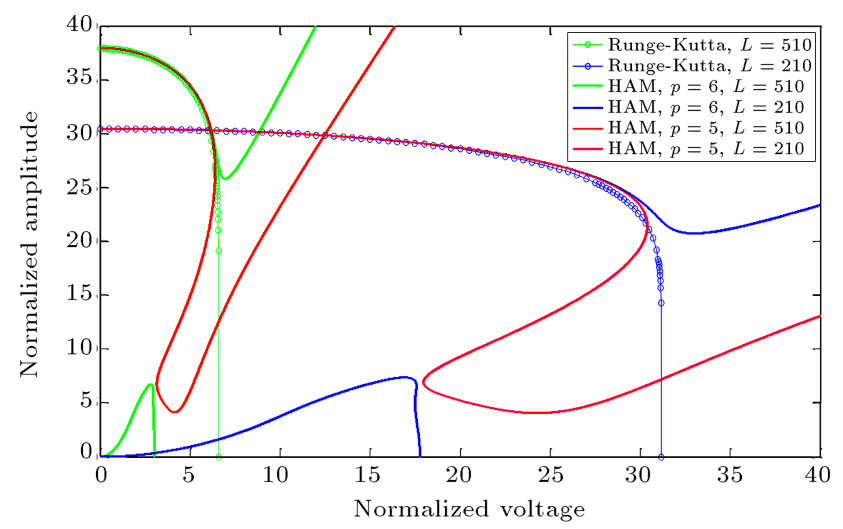

Figure 8. Variations of the normalized nonlinear frequency with applied step voltage $(\mathrm{V})$ for the double-clamped microbeam with length $L=210 \mu \mathrm{m}$ and $L=510 \mu \mathrm{m}$ in different values of $p$.

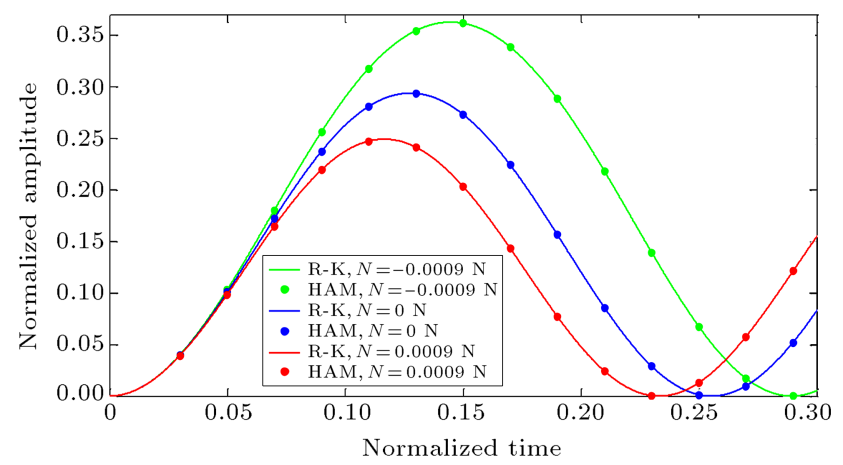

Figure 9. Midpoint-deflection-time history for the double-clamped microbeam with length $L=210 \mu \mathrm{m}$ and different residual axial loads actuated by input voltage of $25 \mathrm{~V}$.

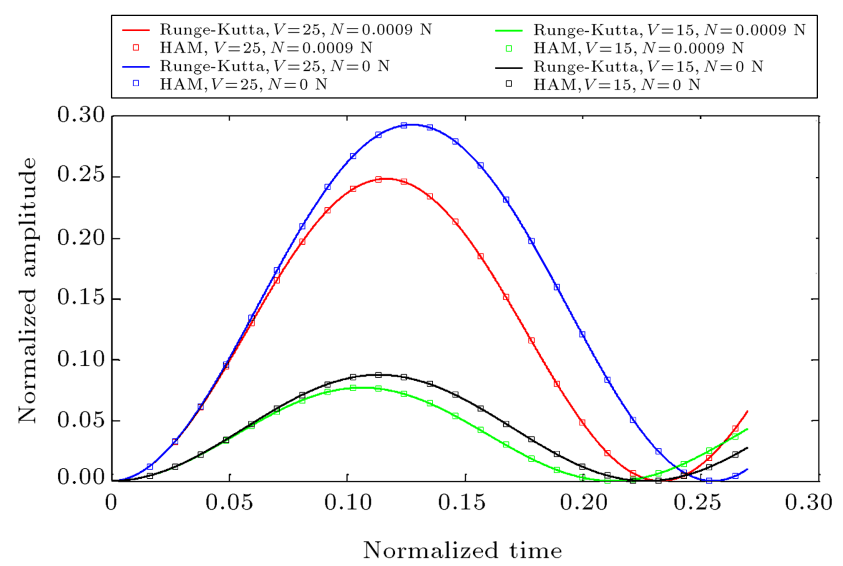

Figure 10. Midpoint deflection time history for the double-clamped microbeam with length $L=210 \mu \mathrm{m}$ $(p=6, m=6)$ for a step change of voltage. that the nonlinear effect of mid-plane stretching is larger at higher applied voltages.

Variations of frequency versus applied voltage using semi-analytic $(p=6)$ and numerical methods, including strain gradient theory for microbeams with length $L=510 \mu \mathrm{m}$ and at different axial loads, are presented in Figure 11. As can be seen, with increasing axial load, frequency and pull-in voltage increase

In the homotopy analysis method, it is easy to find a proper value of $\hbar$ from the $\hbar$-frequency curves to ensure that the solution series converge [36]. These curves ( $\hbar$-curves) depict variations of solutions with $\hbar$. The convergence region and rate of the solution series can be adjusted by means of auxiliary parameter $\hbar$. In this way, $\hbar$ should be selected from the area where the slope of $\hbar$-curves is zero. A proper solution series has to be independent of auxiliary parameter $\hbar$. The $\hbar$ curve of normalized frequency for the microbeam with length $L=510 \mu \mathrm{m}$ is depicted in Figures 12 and 13 . The microbeam is actuated by step voltages of 5.5 and $6.2 \mathrm{~V}$.

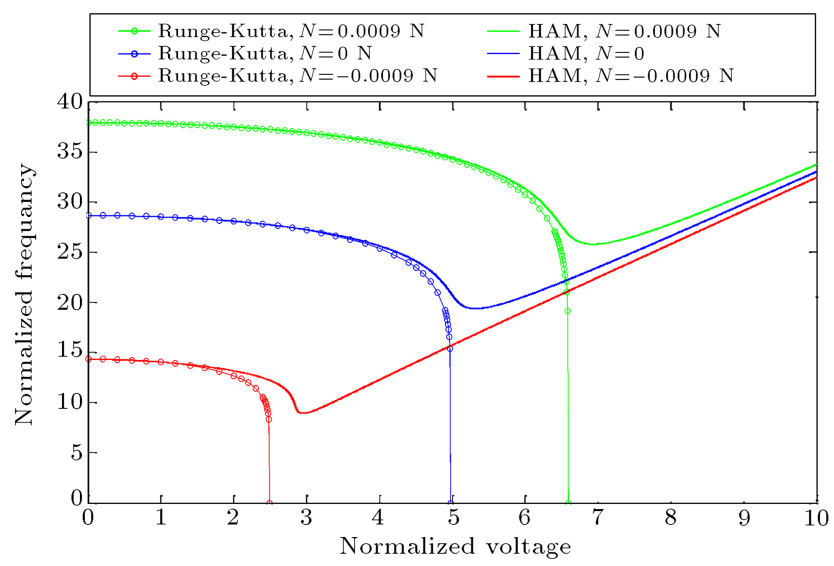

Figure 11. Variations of the normalized nonlinear frequency with applied step voltage $(\mathrm{V})$ for the double-clamped microbeam with length $L=510 \mu \mathrm{m}$ in different axial loads.

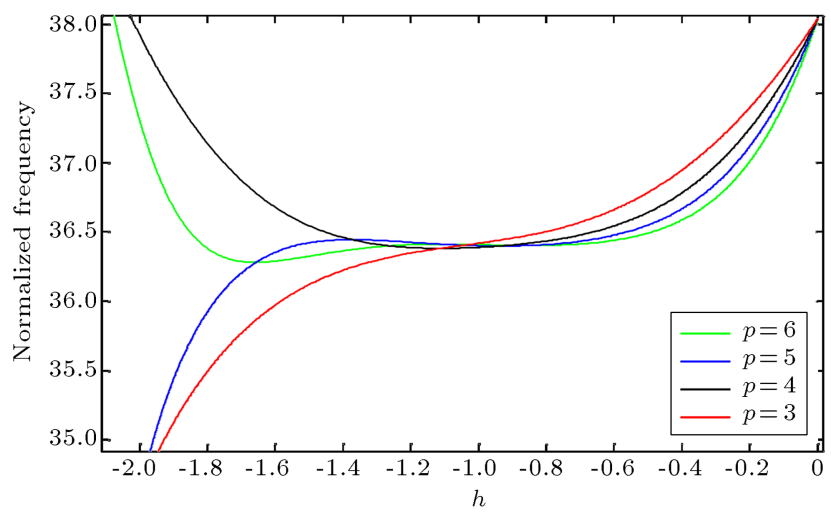

Figure 12. The $\hbar$-curve of normalized frequency for the microbeam with length $L=510 \mu \mathrm{m}$ actuated by a $5.5 \mathrm{~V}$ step-input voltage. 


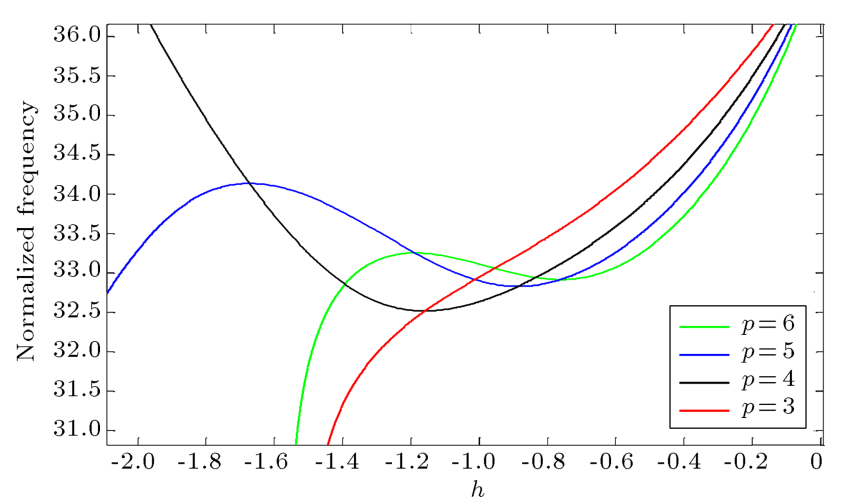

Figure 13. The $\hbar$-curve of normalized frequency for the microbeam with length $L=510 \mu$ m actuated by a $6.2 \mathrm{~V}$ step-input voltage.

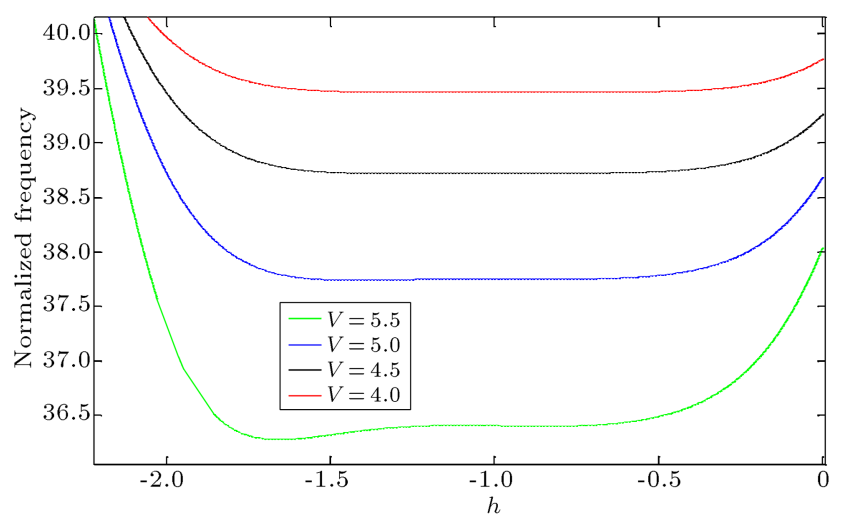

Figure 14. The $\hbar$-curve of normalized frequency for the microbeam with length $L=510 \mu \mathrm{m}$ actuated by different voltages $(p=6)$.

In Figure 12, it is shown that at voltages lower than the pull-in voltage, good agreement results. It is clear that at low voltage with different $p$ approximations, one $\hbar$ value can be achieved.

In Figure 13, it is shown that at voltages close to the pull-in voltage $\left(\mathrm{V}_{p}=6.58491485\right)$, the convergence regions become smaller, and a particular $\hbar$ value is needed for each value of approximation $p$.

From Figures 12 and 13, it can be concluded that using the higher orders of approximation $(p)$ enlarges the convergence regions of the solution series.

In Figures 14 and 15, normalized $\hbar$-frequency curves at different voltages for $p=6$ are presented. From Figures 14 and 15, it is evident that at voltages smaller than the pull-in voltage, an equivalent $\hbar$ value is achieved. At voltages close to pull-in voltage, the convergence domain tends to be smaller; for all approximations, $\hbar$ must be calculated separately for each voltage.

The $\hbar$-curve of normalized frequency for the microbeam with length $L=510 \mu \mathrm{m}$ that is actuated by a step voltage of $5.5 \mathrm{~V}$ with $p=6$ is shown in Figure 16 .

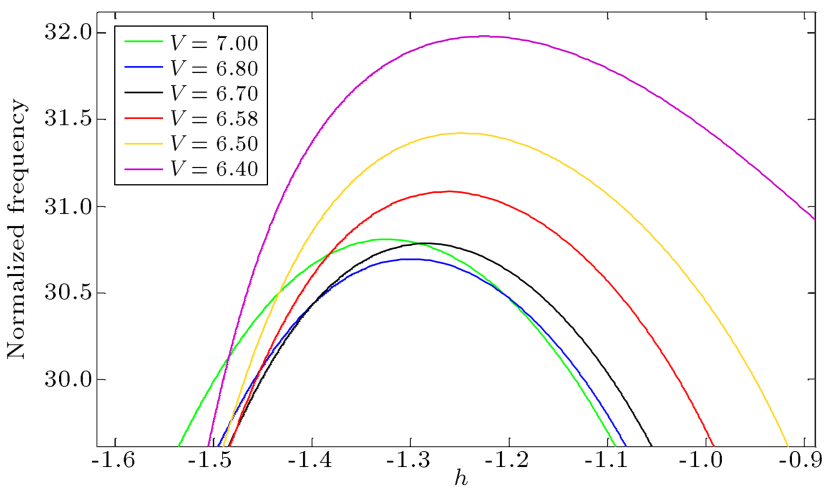

Figure 15. The $\hbar$-curve of normalized frequency for the microbeam with length $L=510 \mu \mathrm{m}$ actuated by different voltages $(p=6)$.

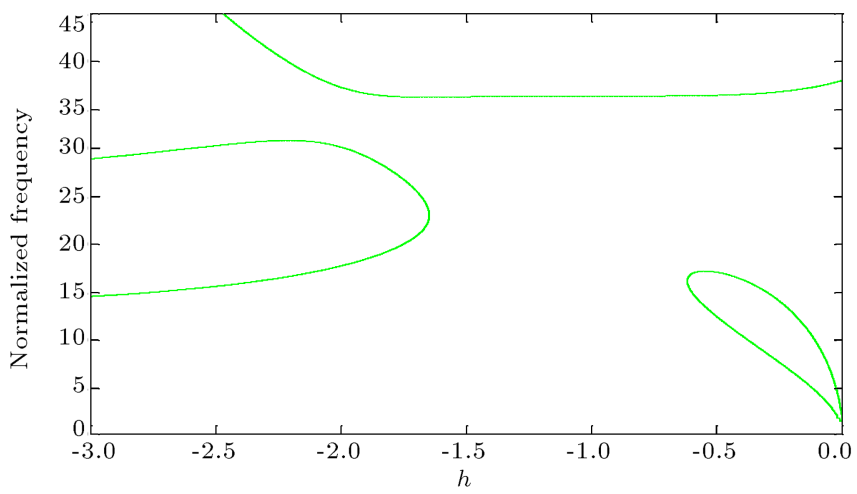

Figure 16. The $\hbar$-curve of normalized frequency for the microbeam with length $L=510 \mu \mathrm{m}$ actuated by a $5.5 \mathrm{~V}$ step-input voltage $(p=6)$.

\section{Conclusion}

Comparing the results of the semi-analytic method with those of numerical technique shows that excellent agreement exists between the figures at voltages smaller than pull-in voltage, while at voltages close to pullin voltage, higher-order approximations are required to find deflections accurately. Effect of auxiliary parameter of HAM is considered to determine convergence criteria. Influence of input voltage and order of HAM approximation on convergence regions is also investigated. Results show that a large convergence region of the solution series exists at low voltages with respect to pull-in voltage, and one $\hbar$ value can be used for various approximations and voltages. When input voltage is chosen to be close to pull-in voltage, convergence regions becomes smaller, so that $\hbar$ must be calculated separately for each order of approximation in any certain input voltage. Also, with increasing order of approximation, convergence regions are larger at certain input voltages.

\section{Acknowledgment}

This paper is supported by INSF. 


\section{References}

1. Batra, R.C., Porfiri, M. and Spinello, D. "Vibrations of narrow microbeams predeformed by an electric field", Journal of Sound and Vibration, 309(3-5), pp. 600-612 (2008).

2. Taylor, G.I. "The coalescence of closely spaced drops when they are at different electric potentials", Proceedings of the Royal Society A, 306(1478), pp. 423-434 (1968).

3. Nathanson, H.C., Newell, W.E., Wickstrom, R.A. and Davis, J.R. "The resonant gate transistor", IEEE Transactions on Electron Devices, 14(3), pp. 117-133 (1967).

4. Osterberg, P.M. "Electrostatically actuated microelectromechanical test structures for material property measurement", PhD Dissertation, Massachusetts Institute of Technology, (1995).

5. Rochus, V., Rixen, D.J. and Golinval, J.C. "Electrostatic coupling of MEMS structures: transient simulations and dynamic pull-in", Nonlinear Analysis: Theory, Methods \& Applications, 63(5-7), pp. 16191633 (2005).

6. Krylov, S. "Lyapunov exponents as a criterion for the dynamic pull-in instability of electrostatically actuated microstructures", International Journal of Nonlinear Mechanics, 42(4), pp. 626-642 (2007).

7. Abdel-Rahman, E.M., Younis, M.I. and Nayfeh, A.H. "Characterization of the mechanical behavior of an electrically actuated microbeam", Journal of Micromechanics and Microengineering, 12(6), pp. 759-766 (2002).

8. De, S.K. and Aluru, N.R. "Complex nonlinear oscillations in electrostatically actuated microstructures", Journal of Microelectromechanical Systems, 15(2), pp. 355-369 (2006).

9. Moghimi Zand, M. and Ahmadian, M.T. "Characterization of coupled-domain multi-layer microplates in pull-in phenomenon, vibrations and dynamics", International Journal of Mechanical Sciences, 49(11), pp. 1226-1237 (2007).

10. Moghimi Zand, M. and Ahmadian, M.T. "Vibrational analysis of electrostatically actuated microstructures considering nonlinear effects", Communications in Nonlinear Science and Numerical Simulation, 14(4) pp. 1664-1678 (2009).

11. Koiter, W.T. "Couple-stresses in the theory of elasticity I and II", Proceedings Koninklijke Nederlandse Akademie van Wetenschappen, Series B; 67 pp. 17-44 (1964).

12. Mindlin, R.D. and Tiersten, H.F. "Effects of couplestresses in linear elasticity", Archive for Rational Mechanics and Analysis, 11(1), pp. 415-448 (1962).

13. Asghari, M., Kahrobaiyan, M.H., Rahaeifard, M. and Ahmadian, M.T. "Investigation of the size effects in Timoshenko beams based on the couple stress theory", Archive of Applied Mechanics, 81(7), pp. 863-874 (2011).
14. Yang, F., Chong, A.C.M., Lam, D.C.C. and Tong, P. "Couple stress based strain gradient theory for elasticity", International Journal of Solids and Structures, 39(10), pp. 2731-2743 (2002).

15. Park, S.K. and Gao, X.L. "Bernoulli-Euler beam model based on a modified couple stress theory", $J$. Micromech. Microeng, 16(11), pp. 2355-2359 (2006).

16. Kong, S., Zhou, S. Nie, Z. and Wang, K. "The sizedependent natural frequency of Bernoulli-Euler microbeams", Int. J. Eng. Sci., 46(5), pp. 427-437 (2008).

17. Ma, H.M., Gao, X.L. and Reddy, J.N. "A microstructure dependent Timoshenko beam model based on a modified couple stress theory", J. Mech. Phys. Solids, 56(12), pp. 3379-3391 (2008).

18. Xia, W., Wang, L. and Yin, L. "Nonlinear nonclassical microscale beams: static bending, postbuckling and free vibration", Int. J. Eng. Sci., 48(12), pp. 20442053 (2010).

19. Asghari, M., Kahrobaiyan, M.H. and Ahmadian, M.T. "A nonlinear Timoshenko beam formulation based on the modified couple stress theory", Int. J. Eng. Sci., 48(12), pp. 1749-1761 (2010).

20. Mindlin, R.D. "Micro-structure in linear elasticity", Archive for Rational Mechanics and Analysis, 16(1), pp. 51-78 (1964).

21. Mindlin, R.D. "Second gradient of strain and surfacetension in linear elasticity", International Journal of Solids and Structures, 1(4), pp. 417-438 (1965).

22. Mindlin, R.D. and Eshel, N.N. "On first straingradient theories in linear elasticity", International Journal of Solids and Structures 4(1), pp. 109-124 (1968).

23. Wang, B., Zhao, J. and Zhou, S. "A micro scale Timoshenko beam model based on strain gradient elasticity theory", European Journal of Mechanics A/Solids, 29(4), pp. 591-599 (2010).

24. Sedighi, H.M. "Size-dependent dynamic pull-in instability of vibrating electrically actuated microbeams based on the strain gradient elasticity theory", Acta Astronautica, 95, pp. 111-123 (2014).

25. Kuang, J.H. and Chen, C.J. "Dynamic characteristics of shaped micro-actuators solved using the differential quadrature method", Journal of Micromechanics and Microengineering, 14(4), pp. 647-655 (2004).

26. Konig, E.R. and Wachutka, G. "Multi-parameter homotopy for the numerical analysis of MEMS", Sensors and Actuators A: Physical, 110(1), pp. 39-51 (2004).

27. Nayfeh, A.H. and Younis, M.I. "A new approach to the modeling and simulation of flexible microstructures under the effect of squeeze film damping", Journal of Micromechanics and Microengineering, 14(2), pp. 170181 (2004).

28. Younis, M.I. "Modeling and simulation of microelectromechanical systems in multi-physics fields", $\mathrm{PhD}$ Dissertation, Virginia Polytechnic Institute and state University (2004). 
29. Liao, S.J. "An approximate solution technique which does not depend upon small parameters: a special example", International Journal of Nonlinear Mechanics, 30(3), pp. 371-380 (1995).

30. Moghimi Zand, M., Ahmadian, M.T. and Rashidian, B. "Semi-analytic solutions to nonlinear vibrations of microbeams under suddenly applied voltages", Journal of Sound and Vibration, 325(1-2), pp. 382-396 (2009).

31. Bao, M. and Yang, H. "Squeeze film air damping in MEMS", Sensors and Actuators A: Physical, 136(1), pp. 3-27 (2007).

32. Rajabi, F. and Ramezani, S. "A nonlinear microbeam model based on strain gradient elasticity theory", Acta Mechanica Solida Sinica, 26(1), pp. 21-34 (2013).

33. He, X.J., Wu, Q., Wang, Y., Song, M.X. and Yin, J.H. "Numerical simulation and analysis of electrically actuated microbeam-based MEMS capacitive switch", Microsystem Technologies, 15, pp. 301-307 (2009).

34. Batra, R.C., Porfiri, M. and Spinello, D. "Capacitance estimate for electrostatically actuated narrow microbeams", IET Micro \& Nano Letters, 1(2), pp. 71-73 (2006).

35. Batra, R.C., Porfiri, M. and Spinello, D. "Electromechanical model of electrically actuated narrow microbeams", Journal of Microelectromechanical Systems, 15(5), pp. 1175-1189 (2006).

36. Liao, S.J., Beyond Perturbation: Introduction to Homotopy Analysis Method, In Chapman \& Hall/CRC, 336 Pages, Boca Raton, London, New York, Washington DC (2004).

37. Liao, S.J. and Cheung, A.T. "Application of homotopy analysis method in nonlinear oscillations", ASME Journal of Applied Mechanics, 65(4), pp. 914-922 (1998).

38. Liao, S.J. "Analytic approximate technique for free oscillations of positively damped systems with algebraically decaying amplitude", International Journal of Nonlinear Mechanics, 38(8), pp. 1173-1183 (2003).

39. Li, S. and Liao, S.J. "Analytic approach to solve multiple solutions of a strongly nonlinear problem", Applied Mathematics and Computation, 169(2), pp. 854-865 (2005).

40. Hayat, T. and Sajid, M. "On analytic solution for thin film flow of a fourth grade fluid down a vertical cylinder", Physics Letter A 361, pp. 316-322 (2007).

41. Abbasbandy, S. "The application of the homotopy analysis method to nonlinear equations arising in heat transfer", Physics Letter A 360(1), pp. 109-113 (2006).

42. Derakhshan, R. "Effect of curved micro-beam on natural frequency and pull-in voltage considering strain gradient theory", M.Sc. Thesis, Sharif University of Technology (2013).

43. Roozbahani, M., Heydarzadeh, N. and Moghimi Zand, M. "Analytical solutions to nonlinear oscillations of a microbeam using higher order beam theory", Scientia Iranica B, 23(5) pp. 2179-2193 (2016).
44. Younis, M. and Nayfeh, A. "A study of the nonlinear response of a resonant microbeam to an electric actuation", Nonlinear Dynamics, 31(1), pp. 91-117 (2003).

45. Mojahedi, M., Moghimi Zand, M. and Ahmadian, M.T "Static pull-in analysis of electrostatically actuated microbeams using homotopy perturbation method", Applied Mathematical Modelling, 34(4), pp. 1032-1041 (2010).

46. Osterberg, P.M. "Electrostatically actuated micromechanical test structure for material property measurement", PhD Dissertation, Massachusetts Institute of Technology; (1995).

\section{Appendix A}

$$
\begin{aligned}
M_{0}= & -\frac{1}{4} \lambda^{2}(1+\gamma \beta) \int_{0}^{1} \phi_{1}(\xi) d \xi \\
M_{1}= & -\frac{1}{4} \lambda^{2}(2+\gamma \beta) \int_{0}^{1} \phi_{1}(\xi)^{2} d \xi \\
& +\left[1+\frac{1}{15} \mu_{s}\left(\frac{30 l_{0}^{2}}{l_{2}^{2}}+\frac{l_{1}^{2}}{l_{2}^{2}}+15\right)\right] \\
& \int_{0}^{1}\left(\frac{\partial^{4}}{\partial \xi^{4}} \phi_{1}(\xi)\right) \phi_{1}(\xi) d \xi \\
& -\frac{1}{30} \frac{h^{2}}{L^{2}} \mu_{s}\left(\frac{5 l_{0}^{2}}{l_{2}^{2}}+\frac{2 l_{1}^{2}}{l_{2}^{2}}\right) \int_{0}^{1}\left(\frac{\partial^{6}}{\partial \xi^{6}} \phi_{1}(\xi)\right) \phi_{1}(\xi) d \xi \\
& -f_{i} \int_{0}^{1}\left(\frac{\partial^{2}}{\partial \xi^{2}} p h i_{1}(\xi)\right) \phi_{1}(\xi) d \xi
\end{aligned}
$$$$
M_{2}=-\frac{1}{4} \lambda^{2}(3+\gamma \beta) \int_{0}^{1} \phi_{1}(\xi)^{3} d \xi
$$$$
M_{3}=-\frac{1}{4} \lambda^{2}(4+\gamma \beta) \int_{0}^{1} \phi_{1}(\xi)^{4} d \xi
$$$$
-\alpha\left[\int_{0}^{1} \phi_{1}(\xi)\left(\frac{d^{2}}{d \xi^{2}} \phi_{1}(\xi)\right)\right.
$$$$
\left.\left(\int_{0}^{1}\left(\frac{d}{d \xi} \phi_{1}(\xi)\right)^{2} d \xi\right) d \xi\right]
$$$$
M_{4}=-\frac{1}{4} \lambda^{2}(5+\gamma \beta) \int_{0}^{1} \phi_{1}(\xi)^{5} d \xi
$$ 


$$
\begin{aligned}
& M_{5}=-\frac{1}{4} \lambda^{2}(6+\gamma \beta) \int_{0}^{1} \phi_{1}(\xi)^{6} d \xi, \\
& M_{6}=-\frac{1}{4} \lambda^{2}(7+\gamma \beta) \int_{0}^{1} \phi_{1}(\xi)^{6} d \xi .
\end{aligned}
$$

\section{Appendix B}

$$
\begin{aligned}
u_{1}(\tau)= & \frac{\hbar M_{0}[1-\cos (\omega \tau)]}{\omega^{2}}, \\
u_{2}(\tau)= & \frac{\hbar M_{0}(\hbar+1)[1-\cos (\omega \tau)]}{\omega^{2}}, \\
u_{3}(\tau)= & -\frac{\hbar M_{0}}{6 \omega^{6}}\left[\left(6 \omega^{4}+12 \hbar \omega^{4}+6 \hbar^{2} \omega^{4}\right.\right. \\
& \left.-4 M_{0} M_{2}\right) \cos (\omega \tau)+\left(M_{0} M_{2} \hbar^{2}\right) \cos (2 \omega \tau) \\
& -6 \hbar^{2} \omega^{4}-12 \hbar \omega^{4}-6 \omega^{4}+3 M_{0} M_{2} \hbar^{2} .
\end{aligned}
$$

\section{Appendix C}

$$
\begin{aligned}
& \omega_{1}(\omega)=-\frac{2 \hbar M_{0} M_{2}}{\omega^{2}}, \\
& \omega_{2}(\omega)=-\frac{\hbar M_{0}}{4 \omega^{4}}\left(8 M_{2} \omega^{2}+8 M_{2} \hbar \omega^{2}+15 M_{0} M_{3} \hbar\right),
\end{aligned}
$$

$$
\begin{aligned}
\omega_{3}(\omega) & =-\frac{\hbar M_{0}}{6 \omega^{6}}\left(12 M_{2} \omega^{4}+24 M_{2} \hbar \omega^{4}+12 M_{2} \hbar^{2} \omega^{4}\right. \\
& +45 M_{3} M_{0} \hbar \omega^{2}+45 M_{3} M_{0} \hbar^{2} \omega^{2}-7 M_{2}^{2} M_{0} \hbar^{2} \\
& \left.+42 M_{4} M_{0}^{2} \hbar^{2}\right) .
\end{aligned}
$$

\section{Biographies}

Reza Derakhshan received his MS degree in Mechanical Engineering from Sharif University of Technology in 2015. At present, he is a PhD student in the School of Mechanical Engineering at Sharif University of Technology, Tehran, Iran.

Mohammad Taghi Ahmadian received his $\mathrm{PhD}$ degree in Mechanical Engineering from University of Kansas in 1986. He served as an Assistant Professor in University of Missouri from 1984 to 1985, and an Assistant Professor in University of Kansas from 1986 to 1987 . He continued his profession similarly at Sharif university of Technology from 1989. At present, he is a Professor in the School of Mechanical Engineering in Sharif University of Technology, Tehran, Iran.

Keykhosrow Firoozbakhsh received his $\mathrm{PhD}$ degree in Biomechanical Engineering (mechanical major) from Wayne State University, Detroit, Michigan, USA (1969-1972), and his Post Doctorate (bone remodeling) from Biomechanics Department, Tulane University, New Orleans, USA (1979-1980). At present, he is a Professor in the School of Mechanical Engineering at Sharif University of Technology, Tehran, Iran. 